\title{
Toward An Exploration of Habituating to Tinnitus: Perspectives on Sensory Gating
}

DOI: $10.3766 /$ jaaa. 18038

\author{
Mehrnaz Mohebbi* \\ Mohammad Farhadi* \\ Ahmad Daneshi* \\ Saeid Mahmoudian*†
}

\begin{abstract}
Background: Some tinnitus participants habituate to their tinnitus, but some others do not and complain of its annoyance so much. It has been suggested that tinnitus is a habituation deficit. Habituation and the ability to ignore a sensory input depend on the normal function of filtering mechanism of sensory gating.

Purpose: The purpose of this study was to compare behavioral aspects of sensory gating in normal and tinnitus participants to search for the reason why some tinnitus participants habituate to their tinnitus but some others do not.
\end{abstract}

Research Design: This investigation was an observational case-control study.

Study Sample: There were 60 tinnitus participants who were categorized into two tinnitus groups (30 compensated tinnitus participants and 30 decompensated tinnitus participants) based on the complaint of tinnitus annoyance, visual analog scale (VAS) for tinnitus loudness, annoyance, and awareness, scores on Tinnitus Questionnaire (TQ), and Tinnitus Handicap Inventory (THI). Also, there were 30 normal hearing participants without tinnitus as the normal control group.

Data Collection and Analysis: Sensory Gating Inventory (SGI), TQ, THI, and VAS was obtained from all participants. THI, TQ, VAS, and SGI total scores and their factors were compared among the groups. Correlations between SGI scores with THI, TQ, and VAS score were calculated.

Results: The results showed that SGI total score and the scores of its four factors were significantly higher in decompensated tinnitus participants compared with compensated tinnitus participants and normal controls. Also, there was a positive correlation between SGI perceptual modulation factor and TQ emotional distress factor and with the VAS for loudness in decompensated tinnitus group.

Conclusions: These results suggested that tinnitus associated with behavioral aspects of sensory gating and decompensated tinnitus may be a result of deficient sensory gating.

Key Words: habituation, questionnaire, sensory gating, tinnitus

Abbreviations: $\mathrm{ADHD}=$ attention-deficit hyperactivity disorder; $\mathrm{ANOVA}=$ analysis of variance; $\mathrm{SD}=$ standard deviation; SGI = Sensory Gating Inventory; THI = Tinnitus Handicap Inventory; TQ = Tinnitus Questionnaire; VAS = visual analog scale

\section{INTRODUCTION}

$\mathrm{T}$ innitus is perception of a sound, usually noiselike or tonal, in absence of an external physical sound source (Møller, 2003). Although tinnitus is not annoying for some participants and they habituate to the tinnitus easily, it is very bothersome for some others and involves them emotionally and psychologically (Andersson, 2002; Kaltenbach, 2006). Tinnitus can be referred to as either middle-ear tinnitus or

*ENT and Head \& Neck Research Center and Department, Hazrat Rasoul Akram Hospital, Iran University of Medical Sciences, Tehran, Iran; $\dagger$ Department of Otorhinolaryngology, Hannover Medical University (MHH), Hannover, Germany

Corresponding author: Ahmad Daneshi, ENT and Head \& Neck Research Center and Department, Hazrat Rasoul Akram Hospital, Iran University of Medical Sciences (IUMS), Tehran, Iran 1445613131; Email: daneshiahmad@gmail.com

This work was funded by the Iran Ministry of Health (MoH), Ph.D. students' research program award, no. 271/7.11.2016, as a part of Ph.D. thesis. 
sensorineural tinnitus. In sensorineural tinnitus, something is establishing abnormal spontaneous nerve activity. As represented in the brain, this might be an increase in activity, synchronous activity across nerve fibers, or an over-representation of some frequency region or combinations of these three (Tyler and Erlandsson, 2003). Sensorineural tinnitus participants are categorized into two groups of compensated and decompensated based on habituating to tinnitus. In compensated tinnitus, participants habituate to the tinnitus and do not complain of its annoyance. In decompensated tinnitus, participants do not habituate to the tinnitus and complain of its annoyance and tinnitus becomes so bothersome that may lead to psychological symptoms such as depression, anxiety, distress, insomnia, aggression, concentration problems, and even suicide (Stobik et al, 2005).

Hallam et al (1988) suggested a habituation theory for tinnitus. According to this theory, suffering and other complaints to tinnitus are consequences of a loss of habituation to the repeating and noninformative sound. Whereas Hallam mentioned only a cognitive habituation failure, others have tried to validate this hypothesis by using different psychophysiological measures with which attention and habituation can be observed (Carlsson and Erlandsson, 1991; Low et al, 2007; Strauss et al, 2008). Studies revealed that tinnitus participants are unable to ignore the sound. This indicates a diminished orienting reaction to auditory signals by tinnitus complainers (Attias et al, 1995).

Habituation and the ability to ignore a sensory input depend on the normal function of sensory gating (Dornhoffer et al, 2006). Sensory gating is defined as the preattentive capability of the brain to recognize significant or novel sensory input and to modulate its sensitivity to incoming stimuli (Braff and Geyer, 1990). It is a filtering mechanism of the central nervous system that prevents irrelevant sensory inputs from entering into higher cortex information processing or to respond when the stimulus changes (Adler et al, 1998). Abnormal function of sensory gating can lead to overloading of information into higher cortex and brain dysfunction. This dysfunction is associated with behavioral disorders and psychotic symptoms (McGhie and Chapman, 1961). Sensory gating and sensory memory has been suggested to be impaired in tinnitus participants (Walpurger et al, 2003; Mahmoudian et al, 2013; Joos et al, 2014). The deficient sensory gating function in thalamus may be responsible for tinnitus phantom percept in distressed participants (De Ridder et al, 2011).

Sensory gating can be measured by electrophysiological P50 paradigm (Cromwell et al, 2008) or by Sensory Gating Inventory (SGI) (Hetrick et al, 2012). SGI assesses clinical and behavioral aspects of sensory gating. It objectifies the perceptual abnormalities and functional and psychosocial consequences of sensory gating.
SGI could confirm anomalies of sensory gating and perceptual inundation in schizophrenia (Micoulaud-Franchi and Vion-Dury, 2013; Micoulaud-Franchi et al, 2014; El-Kaim et al, 2015), attention-deficit hyperactivity disorder (ADHD) (Sable et al, 2012; Micoulaud-Franchi et al, 2014; 2016) and Tourette syndrome (Sutherland Owens et al, 2011).

In this study with perspective to behavioral aspects of sensory gating, we looked for answering this question that why some tinnitus participants habituate to their tinnitus but some others do not habituate to it and complain so much of its annoyance. Because tinnitus is a habituation deficit according to Hallam's theory and sensory gating is necessary for habituation, we hypothesized that behavioral aspects of sensory gating may defect in decompensated tinnitus participants compared with compensated tinnitus participants and normal controls. To answer this question, SGI was compared among the normal control and compensated and decompensated tinnitus groups. Then, the correlation of SGI with Tinnitus Handicap Inventory (THI) and Tinnitus Questionnaire (TQ) was computed to determine the relationship of SGI with tinnitus. The aim of this study was to assess behavioral aspects of sensory gating in normal and tinnitus groups to determine the effect of sensory gating on habituating to tinnitus.

\section{MATERIALS AND METHODS}

\section{Participants}

Participants consisted of three groups: 30 compensated tinnitus participants, 30 decompensated tinnitus participants, and 30 normal hearing without tinnitus as the control. They were all native Persian speaking and right handed as shown by the Edinburgh Handedness Inventory (Oldfield, 1971) who voluntarily participated in the study. They were between 18 and 59 years old and had no current or past substance abuse or dependency, no neurological illness, no brain injury, and no auditory impairment. Tinnitus participants had their tinnitus for more than six months and were referred by the ear-nose-throat clinic of the affiliated hospital. Compensated tinnitus participants were included according to the following factors: no complaint of tinnitus annoyance; visual analog scale (VAS) $<3$ for tinnitus loudness, annoyance, and awareness; and scores of $<16$ in THI and $<30$ in TQ. Decompensated tinnitus participants were included according to the following factors: sever complaint of tinnitus annoyance; VAS $>7$ for tinnitus loudness, annoyance, and awareness; and scores of $>58$ in THI and $>60$ in TQ. Construction of VAS was consisted of a $10-\mathrm{cm}$ line with a resolution of 1 in 10 parts and each part was labeled with numbers from 0 to 10 . There were three VASs for loudness, annoyance, and awareness of tinnitus, each labeled on number 0,5 , 
and 10, respectively, as least, moderate, and the most. Participants filled the validated Persian Version of SGI (Mohebbi et al, accepted in Basic and Clinical Neuroscience Journal). Filled questionnaires were checked to ensure that participants did not miss any items to answer. The study procedure was in accordance with the Declaration of Helsinki.

\section{SGI}

SGI is a self-report questionnaire that is composed of 36 items. It addresses perceptual abnormalities related to sensory gating deficit. Each item is scored based on a six-point Likert scale (from $0=$ never true to $5=$ always true). The items are categorized into four factors that have been revealed to play a part in sensory gating: perceptual modulation, distractibility, overinclusion, and fatigue-stress modulation. The Persian version of SGI was used in this study, which has high validity and reliability (Mohebbi et al, accepted in Basic and Clinical Neuroscience Journal). The algebraic sum of Likert scale for items was calculated to obtain the overall score of SGI and the scores for each factor.

\section{Statistical Analyses}

Data were analyzed using SPSS software (version 21, PASW Statistics; IBM Corporation, NY). Demographic characteristics were compared among the groups using a $t$-test or a one-way analysis of variance (ANOVA). Mean and standard deviations (SDs) for SGI total score and scores of SGI factors were calculated in all groups and they were compared among the three studied groups using a Kruskal-Wallis test. Dunn's test was performed to determine group differences. A Mann-Whitney test was performed to compare scores of THI, TQ, and VAS for loudness, annoyance, and awareness between compensated and decompensated tinnitus groups. Spearman's correlation coefficient was determined to test the relationship between SGI scores with VAS, THI, and TQ scores.

\section{RESULTS}

\section{Demographic Characteristics}

A total of 90 participants filled the SGI. They consisted of 48 males and 42 females. The mean age had no significant differences among the three studied groups as shown in One-way ANOVA test $\left[F_{(2,87)}=0.17, p>\right.$ 0.05]. Also, one-way ANOVA test for hearing thresholds in frequencies of $250,500,1000,2000,4000,6000$, and $8000 \mathrm{~Hz}$ did not show significant differences among the three groups $\left[F_{(2,87)}=0.17, p>0.05\right]$. Tinnitus duration, pitch match of tinnitus, and loudness match of tinnitus were not significantly different between compensated and decompensated tinnitus group as indicated by the $t$-test (Table 1). A Mann-Whitney test was performed to compare scores for THI, TQ, and VAS for loudness, VAS for annoyance, and VAS for awareness between compensated and decompensated tinnitus groups. It showed that THI, TQ, and VAS scores were significantly higher in decompensated tinnitus group compared with compensated tinnitus group (Table 2).

\section{SGI Comparisons among the Groups}

A Kruskal-Wallis test was conducted to compare the effect of tinnitus on SGI total score and scores of its four

Table 1. Demographic Properties of the Studied Groups

\begin{tabular}{|c|c|c|c|c|c|c|}
\hline & Normal Control & Compensated Tinnitus & Decompensated Tinnitus & $F$ & $t$ & $p$ \\
\hline$\overline{\mathrm{Age}}$ & $41.50 \pm 11.95$ & $43.06 \pm 11.24$ & $42.93 \pm 10.99$ & 0.17 & - & 0.84 \\
\hline Male & 15 & 14 & 16 & - & - & - \\
\hline Female & 15 & 16 & 14 & & & \\
\hline \multicolumn{7}{|l|}{ Pure-tone thresholds } \\
\hline 250 & $10.83 \pm 7.20$ & $12.50 \pm 7.62$ & $11.00 \pm 6.74$ & 0.48 & - & 0.61 \\
\hline 500 & $13.50 \pm 8.72$ & $14.33 \pm 8.97$ & $10.66 \pm 5.97$ & 1.72 & & 0.18 \\
\hline 1000 & $17.33 \pm 10.64$ & $14.33 \pm 8.74$ & $13.16 \pm 6.08$ & 1.96 & & 0.14 \\
\hline 2000 & $19.50 \pm 12.41$ & $18.50 \pm 11.82$ & $15.00 \pm 7.07$ & 1.46 & & 0.23 \\
\hline 4000 & $17.50 \pm 10.96$ & $17.33 \pm 12.84$ & $17.83 \pm 9.62$ & 0.01 & & 0.98 \\
\hline 6000 & $18.66 \pm 11.59$ & $19.00 \pm 9.13$ & $21.83 \pm 11.02$ & 0.80 & & 0.45 \\
\hline 8000 & $18.50 \pm 13.71$ & $17.66 \pm 10.56$ & $22.00 \pm 13.74$ & 0.97 & & 0.38 \\
\hline \multicolumn{7}{|l|}{ Tinnitus laterality } \\
\hline Right & - & 10 & 5 & - & - & - \\
\hline Left & & 9 & 10 & & & \\
\hline Bilateral & & 11 & 15 & & & \\
\hline Tinnitus duration & - & $53.43 \pm 71.12$ & $72.93 \pm 80.74$ & - & -0.99 & 0.32 \\
\hline Pitch match of tinnitus & - & $7.5 \pm 1.42$ & $7.6 \pm 1.43$ & & 0.27 & 0.78 \\
\hline Loudness match of tinnitus & - & $6.36 \pm 2.78$ & $7.10 \pm 3.10$ & - & -0.96 & 0.33 \\
\hline
\end{tabular}


factors at $p<0.05$ level in the three studied groups. There was a significant effect of tinnitus on SGI total score and on scores of all four factors for the three groups (Table 3). Multiple comparisons using Dunn's test indicated that the mean SGI total score for the decompensated tinnitus group $(M=100.13$, SD $=$ 18.65) was significantly higher than the normal control group $(M=37.13, \mathrm{SD}=14.54)$ and the compensated tinnitus group $(M=42.53, \mathrm{SD}=22.03)$. The mean score for the factor of perceptual modulation in decompensated tinnitus group $(M=39.53, \mathrm{SD}=10.43)$ was significantly higher than compensated tinnitus group $(M=$ 16.20, $\mathrm{SD}=9.25)$ and normal controls $(M=17.93, \mathrm{SD}=$ 7.48). The mean score for the factor of distractibility in decompensated tinnitus group $(M=24.63, \mathrm{SD}=5.76)$ was significantly higher than compensated tinnitus group $(M=10.23, \mathrm{SD}=7.74)$ and normal controls $(M=8.50, \mathrm{SD}=4.24)$. The mean score for the factor of overinclusion in decompensated tinnitus group $(M=$ 19.60 , SD $=6.19$ ) was significantly higher than compensated tinnitus group $(M=9.90, \mathrm{SD}=5.58)$ and normal controls $(M=6.80, \mathrm{SD}=3.06)$. The mean score for the factor of fatigue-stress modulation in decompensated tinnitus group ( $M=16.36, \mathrm{SD}=3.62)$ was significantly higher than compensated tinnitus group $(M=$ $6.56, \mathrm{SD}=4.36)$ and normal controls $(M=3.90, \mathrm{SD}=$ 2.30 ). Taken together, these results suggest that tinnitus associates with behavioral aspects of sensory gating. Figure 1 shows the results of the multiple comparisons.

\section{SGI Correlations with VAS, THI, and TQ}

Spearman's correlation coefficient was computed to assess the relationship between SGI scores with VAS,
THI, and TQ total scores and their factors in decompensated tinnitus groups. There was a positive correlation between SGI perceptual modulation factor and TQ emotional distress factors $(r=0.44, \mathrm{n}=30, p=0.01)$ and with VAS score for loudness $(r=0.45, \mathrm{n}=30, p=0.01$ ) in decompensated tinnitus group. These findings suggest that the SGI is relevant to tinnitus. As the SGI scores did not showed significant differences between compensated tinnitus group and normal controls, correlations of SGI scores with VAS, THI, and TQ scores were not calculated in the compensated group.

\section{DISCUSSION}

$T$ he present study compared the SGI scores among compensated and decompensated tinnitus participants and normal controls. The results support our hypothesis that decompensated tinnitus participants have a deficit in behavioral aspects of sensory gating compared with compensated tinnitus participants and normal controls. It showed that decompensated tinnitus group with high scores on THI, TQ, and VAS had higher scores on SGI compared with compensated tinnitus group with low scores on THI, TQ, and VAS. Also, compensated tinnitus group reported low scores on SGI total scores and three of SGI factors similar to the normal control group. These results suggest that sensory gating may be a factor in preventing habituation to tinnitus.

\section{SGI Comparisons among the Groups}

Studies revealed sensory gating deficit in ADHD, schizophrenia, and Tourette syndrome using SGI (Sutherland Owens et al, 2011; Sable et al, 2012; Micoulaud-Franchi et al, 2014; 2016). Consistent with

Table 2. Mann-Whitney Test Comparing TQs between the Tinnitus Groups

\begin{tabular}{|c|c|c|c|c|}
\hline & $\begin{array}{c}\text { Compensated Tinnitus } \\
\text { Mean } \pm \text { SD }\end{array}$ & $\begin{array}{c}\text { Decompensated Tinnitus } \\
\text { Mean } \pm \text { SD }\end{array}$ & $Z$ & $p$ \\
\hline$\overline{\mathrm{THI}}$ & $20.03 \pm 6.53$ & $72.46 \pm 12.80$ & -6.65 & 0.00 \\
\hline $\mathrm{TQ}$ & $27.33 \pm 5.24$ & $66.23 \pm 10.85$ & -6.65 & 0.00 \\
\hline VAS_loudness & $2.36 \pm 0.80$ & $7.93 \pm 1.31$ & -6.73 & 0.00 \\
\hline VAS_annoyance & $2.06 \pm 0.86$ & $8.40 \pm 1.30$ & -6.73 & 0.00 \\
\hline VAS_awareness & $1.93 \pm 0.69$ & $8.53 \pm 1.16$ & -6.75 & 0.00 \\
\hline
\end{tabular}

Table 3. Kruskal-Wallis Results Comparing SGI Scores among the Studied Groups

\begin{tabular}{|c|c|c|c|c|c|}
\hline SGI Scores & $\begin{array}{c}\text { Normal Control } \\
\text { Mean } \pm \text { SD }\end{array}$ & $\begin{array}{c}\text { Compensated Tinnitus } \\
\text { Mean } \pm \mathrm{SD}\end{array}$ & $\begin{array}{c}\text { Decompensated Tinnitus } \\
\text { Mean } \pm S D\end{array}$ & $H(2)$ & $p$ \\
\hline Perceptual modulation & $17.93 \pm 7.48$ & $16.20 \pm 9.25$ & $39.53 \pm 10.43$ & 46.87 & 0.00 \\
\hline Overinclusion & $8.50 \pm 4.24$ & $10.23 \pm 7.74$ & $24.63 \pm 5.76$ & 48.87 & 0.00 \\
\hline Distractibility & $6.60 \pm 3.06$ & $9.90 \pm 5.58$ & $19.60 \pm 6.19$ & 42.75 & 0.00 \\
\hline Fatigue-stress modulation & $3.90 \pm 2.30$ & $6.56 \pm 4.36$ & $16.36 \pm 3.62$ & 57.32 & 0.00 \\
\hline Total & $37.13 \pm 14.54$ & $42.53 \pm 22.03$ & $100.13 \pm 18.65$ & 56.55 & 0.00 \\
\hline
\end{tabular}




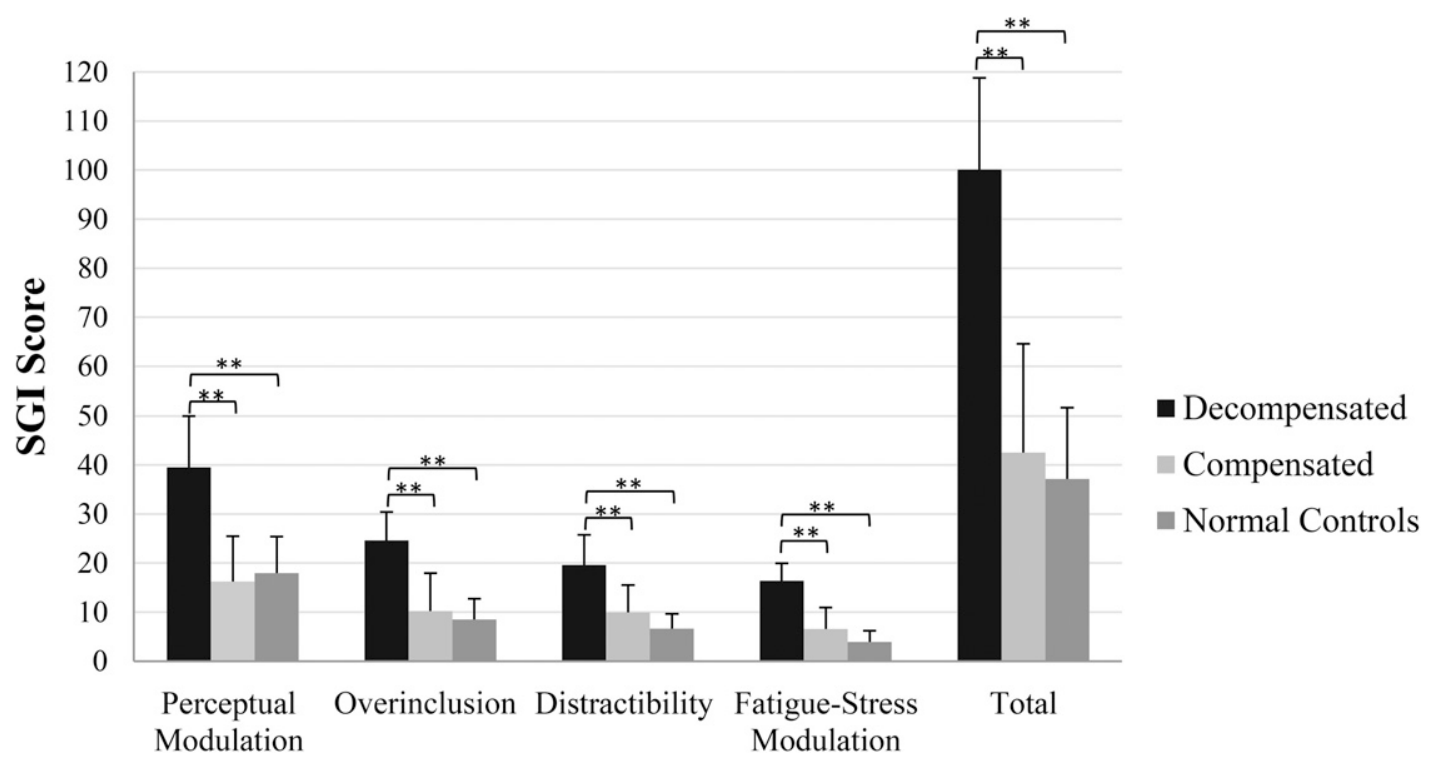

Figure 1. Multiple comparisons using Dunn's test to show differences in SGI scores among the studied groups. ${ }^{*} p<0.05, * * p<0.01$.

our study, they reported higher SGI scores in these abnormalities compared with normal participants. To our knowledge, this is the first study using SGI in tinnitus. ADHD, schizophrenia, and tinnitus share several clinical characteristics (Mcghie and Chapman, 1961). Most of them exhibit sensory over-responsivity and sensory modulation disorder (Yochman et al, 2006) and report being flooded with sensory stimuli (Biederman, 2005). Tinnitus participants report hypersensitivity to sound as a loudness perception disorder called hyperacusis (Tyler and Conrad-Armes, 1983; Baguley, 2003; Pienkowski et al, 2014; Tyler et al, 2014). They frequently report poor mental concentration (Cuny et al, 2004). Because of these common clinical characteristics, there may be common mechanisms responsible for the sensory modulation disorder. The SGI may be detecting a broad sensory gating deficiency in these abnormalities.

The hyperacusis can be determined by hyperacusis questionnaire (Khalfa et al, 2002). Tinnitus and hyperacusis can coexist. These symptoms of excessive auditory perception often share the same psychological profile and common mechanisms may be responsible for them (Meric et al, 1998; Vendrig, 2000). Some studies suggest that hippocampus, amygdale, and somatosensory system may also be involved in tinnitus and hyperacusis (De Ridder et al, 2006; Hwang et al, 2009). Given the overlap between tinnitus and hyperacusis mechanisms, it is important to discuss hyperacusis when assessing models of tinnitus (Knipper et al, 2013).

Reduced input to the brain caused by deafferentation or an imbalanced state in the brain increases the cen- tral noise, generating tinnitus. It leads to an increased nonlinear gain in the form of steepened loudness growth as either loudness recruitment or hyperacusis (Zeng, 2013). Sensory gating deficits may be related to the impaired inhibitory circuitry and the imbalance of the excitatory and inhibitory in the central auditory system. It also may associate with the impaired temporal processing and altered temporal integration (Rauschecker et al, 2015). With increasing duration of a stimulus, temporal integration manifests as a decrease in threshold or increased strength of the perception of a stimulus (Møller, 1999).

In our study, participants with decompensated tinnitus reported SGI scores higher than the compensated tinnitus participants on all four SGI factors. Consistent with our results, sensory gating deficit in tinnitus was reported in previous studies using different assessment techniques. Based on structural and functional changes, it has been assumed that tinnitus is the result of a deficient thalamic sensory attention gating mechanism (Rauschecker et al, 2010; Leaver et al, 2011; Joos et al, 2014).

Among electrophysiology studies, Walpurger et al (2003) showed that N1 response, a marker for late sensory gating, was less decreased when repetitive auditory stimuli were presented in tinnitus participants. They concluded that there is less habituation to tinnitus. By contrast, Dornhoffer et al (2006) showed no significant differences in P50 sensory gating as measured by the ability to suppress a second, closely paired stimulus. They suggested that tinnitus patients had no impairment in sensory gating and the fundamental deficit 
in habituation appeared not to be at the brainstemthalamus level. They concluded that patients with tinnitus had a normal ability to habituate to externally generated repetitive stimuli.

Moreover, relatively higher level neurophysiological processing could be related to sensory gating, which may be shown by higher processing electrophysiological responses such as N100, P200, or P300. N100 is an index of sound detection and is associated with attention properties. N100, combined with the P200 component, also known as N1-P2, represents the late phase of sensory gating. Sensory memory deficit was reported in tinnitus participants by auditory mismatch negativity (Weisz et al, 2004; Mahmoudian et al, 2013), a mechanism needed for normal function of sensory gating (Kisley et al, 2004; Lijffijt et al, 2009). Significantly smaller N1 amplitude was demonstrated in tinnitus patients than those obtained from normal participants (Jacobson and McCaslin, 2003). Larger P1 amplitude in the study of Konadath and Manjula (2016) was attributed to the mechanism explaining central gain model. Considering these evidence, it is suggested that abnormalities in later components can be a matter of concern.

There are many theories about tinnitus, which none of them is accepted universally, but most propose a central nervous system dysfunction for it. Hallam et al (1988) proposed that tinnitus is a habituation deficit wherein the tinnitus sound continuously elicits unattenuated orienting responses, thus demanding constant attention. The process of habituation is a consequence of sensory gating (Dornhoffer et al, 2006). Sensory gating is a critical mechanism to focus attention and filter out irrelevant sensory input. It has been hypothesized that tinnitus is the result of a deficient sensory attention gating mechanism (Rauschecker et al, 2010; Leaver et al, 2011). The involvement of the parahippocampus in tinnitus which has a sensory gating function might confirm our results. Constant updating of tinnitus percept from memory as a result of deficient sensory memory and sensory gating prevent habituation (De Ridder et al, 2006).

\section{SGI Correlations with VAS, THI, and TQ}

To our knowledge, this is the first study which evaluated the relationship between SGI factors with TQ, THI, and VAS. The positive correlation between perceptual modulation factor of SGI with the emotional distress of TQ and VAS for loudness may indicate that sensory gating has the most impact on emotional distress and loudness of tinnitus. Tinnitus perception has been found to be strongly correlated with emotional impact (Jastreboff and Azell, 1993; Lim et al, 2010). We propose that the perceptual modulation may be the SGI factor which correlates best with tin- nitus. Also, emotional distress factor of $\mathrm{TQ}$ relates best to SGI and can be a predictor of sensory gating deficit. This may be associated with the items of these two factors. Lack of relationship between SGI and THI in decompensated tinnitus participants may be due to this reason that SGI is not specifically designed for tinnitus. Moreover, SGI has some items other than auditory modality, which may affect the relationship between SGI and questionnaires related to tinnitus. Considering these, SGI should be used with caution in tinnitus. We propose to use auditory items of SGI in tinnitus.

\section{Limitations}

This study was limited by the small sample size, probable comorbid psychological conditions, and lack of an objective method for correlating with SGI. However, our vigorous findings suggest that the SGI as an index of sensory gating could reveal preliminary information about differences between compensated and decompensated tinnitus participants. These data may lead toward answering the question why some participants habituate to tinnitus and why others do not.

\section{CONCLUSIONS}

$T$ his study suggested the SGI as a simple and rapid preliminary screening tool for the presence or absence of sensory gating in tinnitus participants. Higher scores of SGI in decompensated tinnitus participants support conceptual models wherein abnormal neurophysiologic mechanisms to repetitive stimuli give rise to dishabituation to sensory stimuli. Further investigations on the relationship between SGI and other assessment techniques such as electrophysiological measures of sensory gating (i.e., N100/P200, MMN, and P300) can contribute valuable information for the psychophysiological impact of sensory gating in tinnitus.

Acknowledgments. We would like to show our gratitude to the personnel in the ENT clinic of Rasool Akram hospital and the audiology clinic of Amir Alam hospital for referring tinnitus participants and providing equipment for performing audiological evaluations.

\section{REFERENCES}

Adler LE, Olincy A, Waldo M, Harris JG, Griffith J, Stevens K, Flach K, Nagamoto H, Bickford P, Leonard S, Freedman R. (1998) Schizophrenia, sensory gating, and nicotinic receptors. Schizophr Bull 24(2):189-202.

Andersson G. (2002) Psychological aspects of tinnitus and the application of cognitive-behavioral therapy. Clin Psychol Rev 22(7): 977-990. 
Attias J, Bresloff I, Furman V, Urbach D. (1995) Auditory event related potentials in simulated tinnitus. $J$ Basic Clin Physiol Pharmacol 6(2):173-184.

Baguley DM. (2003) Hyperacusis. J R Soc Med 96(12):582-585.

Biederman J. (2005) Attention-deficit/hyperactivity disorder: a selective overview. Biol Psychiatry 57(11):1215-1220.

Braff DL, Geyer MA. (1990) Sensorimotor gating and schizophrenia. Human and animal model studies. Arch Gen Psychiatr 47(2): 181-188.

Carlsson SG, Erlandsson SI. (1991) Habituation and tinnitus: an experimental study. J Psychosom Res 35(4-5):509-514.

Cromwell HC, Mears RP, Wan L, Boutros NN. (2008) Sensory gating: a translational effort from basic to clinical science. Clin EEG Neurosci 39(2):69-72.

Cuny C, Norena A, El Massioui F, Chéry-Croze S. (2004) Reduced attention shift in response to auditory changes in subjects with tinnitus. Audiol and Neurotol 9(5):294-302.

De Ridder D, Elgoyhen AB, Romo R, Langguth B. (2011) Phantom percepts: tinnitus and pain as persisting aversive memory networks. Proc Natl Acad Sci 108(20):8075-8080.

De Ridder D, Fransen H, Francois O, Sunaert S, Kovacs S, Van De Heyning P. (2006) Amygdalohippocampal involvement in tinnitus and auditory memory. Acta Otolaryngol Suppl 126(556):50-53.

Dornhoffer J, Danner C, Mennemeier M, Blake D, Garcia-Rill E. (2006) Arousal and attention deficits in patients with tinnitus. Int Tinnitus $J$ 12(1):9-16.

El-Kaim A, Aramaki M, Ystad S, Kronland-Martinet R, Cermolacce M, Naudin J, Vion-Dury J, Micoulaud-Franchi J-A. (2015) On the correlation between perceptual inundation caused by realistic immersive environmental auditory scenes and the sensory gating inventory in schizophrenia. Eur Psychiatry $J$ Assoc Eur Psychiatrists 30(5):606-614.

Hallam RS, Jakes SC, Hinchcliffe R. (1988) Cognitive variables in tinnitus annoyance. Br J Clin Psychol 27(3):213-222.

Hetrick WP, Erickson MA, Smith DA. (2012) Phenomenological dimensions of sensory gating. Schizophr Bull 38(1):178-191.

Hwang JH, Chou PH, Wu CW, Chen JH, Liu TC. (2009) Brain activation in patients with idiopathic hyperacusis. Am J Otolaryngol 30(6):432-434.

Jacobson GP, McCaslin DL. (2003) A reexamination of the long latency $\mathrm{N} 1$ response in patients with tinnitus. J Am Acad Audiol 14(7):393-400.

Jastreboff PJ, Azell JWP. (1993) A neurophysiological approach to tinnitus: clinical implications. Br J Audiol 27(1):7-17.

Joos K, Gilles A, Van de Heyning P, De Ridder D, Vanneste S. (2014) From sensation to percept: the neural signature of auditory event-related potentials. Neurosci Biobehav Rev 42: 148-156.

Kaltenbach JA. (2006) The dorsal cochlear nucleus as a participant in the auditory, attentional and emotional components of tinnitus. Hear Res 216-217:224-234.

Khalfa S, Dubal S, Veuillet E, Perez-Diaz F, Jouvent R, Collet L. (2002) Psychometric normalization of a hyperacusis questionnaire. ORL J Otorhinolaryngol Relat Spec 64(6):436-442.
Kisley MA, Noecker TL, Guinther PM. (2004) Comparison of sensory gating to mismatch negativity and self-reported perceptual phenomena in healthy adults. Psychophysiology 41(4): 604-612.

Knipper M, Van Dijk P, Nunes I, Rüttiger L, Zimmermann U. (2013) Advances in the neurobiology of hearing disorders: recent developments regarding the basis of tinnitus and hyperacusis. Prog Neurobiol 111:17-33.

Konadath S, Manjula P. (2016) Auditory brainstem response and late latency response in individuals with tinnitus having normal hearing. Intract Rare Dis Res 5(4):262-268.

Leaver AM, Renier L, Chevillet MA, Morgan S, Kim HJ, Rauschecker JP. (2011) Dysregulation of limbic and auditory networks in tinnitus. Neuron 69(1):33-43.

Lijffijt M, Lane SD, Meier SL, Boutros NN, Burroughs S, Steinberg JL, Gerard Moeller F, Swann AC. (2009) P50, N100, and P200 sensory gating: relationships with behavioral inhibition, attention, and working memory. Psychophysiology 46(5): $1059-1068$.

Lim JJBH, Lu PKS, Koh DSQ, Eng SP. (2010) Impact of tinnitus as measured by the Tinnitus Handicap inventory among tinnitus sufferers in Singapore. Singapore Med J 51(7):551-557.

Low YF, Trenado C, Delb W, Corona-Strauss FI, Strauss DJ. (2007) The role of attention in the tinnitus decompensation: reinforcement of a large-scale neural decompensation measure. Conf Proc IEEE Eng Med Biol Soc 2007:2485-2488.

Mahmoudian S, Farhadi M, Najafi-Koopaie M, DarestaniFarahani E, Mohebbi M, Dengler R, Esser KH, Sadjedi H, Salamat B, Danesh AA, Lenarz T. (2013) Central auditory processing during chronic tinnitus as indexed by topographical maps of the mismatch negativity obtained with the multi-feature paradigm. Brain Res 1527:161-173.

McGhie A, Chapman J. (1961) Disorders of attention and perception in early schizophrenia. Br J Med Psychol 34(2):103-116.

Meric C, Gartner M, Collet L, Chéry-Croze S. (1998) Psychopathological profile of tinnitus sufferers: evidence concerning the relationship between tinnitus features and impact on life. Audiol Neuro Otol 3(4):240-252.

Micoulaud-Franchi JA, Vion-Dury J. (2013) What is sensory inundation in schizophrenia? Clin Neurophysiol 124(3):628-629.

Micoulaud-Franchi J-A, Hetrick WP, Aramaki M, Bolbecker A, Boyer L, Ystad S, Kronland-Martinet R, Richieri R, Faget C, Faugere M, El-Kaim A, Cermolacce M, Lancon C, Vion-Dury J. (2014) Do schizophrenia patients with low P50-suppression report more perceptual anomalies with the sensory gating inventory? Schizophr Res 157(1-3):157-162.

Micoulaud-Franchi JA, Lopez R, Cermolacce M, Vaillant F, Péri P, Boyer L, Richieri R, Bioulac S, Sagaspe P, Philip P, Vion-Dury J, Vion-Dury J. (2016) Sensory gating capacity and attentional function in adults with ADHD: a preliminary neurophysiological and neuropsychological study. J Atten Disord 1087054716629716.

Møler AR. (1999, September) Pathophysiology of severe tinnitus and chronic pain. In: Sixth International Tinnitus Seminar (p. 26).

Møller AR. (2003) Pathophysiology of tinnitus. Otolaryngol Clin North Am 36(2):249-266.

Oldfield RC. (1971) The assessment and analysis of handedness: the Edinburgh inventory. Neuropsychologia 9(1):97-113. 
Pienkowski M, Tyler RS, Roncancio ER, Jun HJ, Brozoski T, Dauman N, Martin N. (2014) A review of hyperacusis and future directions: part II. Measurement, mechanisms, and treatment. Am J Audiol 23(4):420-436.

Rauschecker JP, Leaver AM, Mühlau M. (2010) Tuning out the noise: limbic-auditory interactions in tinnitus. Neuron 66(6): $819-826$

Rauschecker JP, May ES, Maudoux A, Ploner M. (2015) Frontostriatal gating of tinnitus and chronic pain. Trends Cogn Sci 19(10): 567-578.

Sable JJ, Kyle MR, Knopf KL, Schully LT, Brooks MM, Parry KH, Diamond RE, Flink LA, Stowe R, Suna E, Thompson IA. (2012) The Sensory Gating Inventory as a potential diagnostic tool for attention-deficit hyperactivity disorder. Atten Defic Hyperact Disord 4(3):141-144.

Stobik C, Weber RK, Münte TF, Walter M, Frommer J. (2005) Evidence of psychosomatic influences in compensated and decompensated tinnitus. Int J Audiol 44(6):370-378.

Strauss DJ, Delb W, D’Amelio R, Low YF, Falkai P. (2008) Objective quantification of the tinnitus decompensation by synchronization measures of auditory evoked single sweeps. Trans Neural Syst Rehabil Eng 16(1):74-81.

Sutherland Owens AN, Miguel EC, Swerdlow NR. (2011) Sensory gating scales and premonitory urges in Tourette syndrome. Sci World J 11:736-741.
Tyler RS, Conrad-Armes D. (1983) The determination of tinnitus loudness considering the effects of recruitment. J Speech Lang Hear Res 26(1):59-72.

Tyler RS, Erlandsson S. (2003) Management of the tinnitus patient. Textbook of Audiological Medicine, 571-578.

Tyler RS, Pienkowski M, Roncancio ER, Jun HJ, Brozoski T, Dauman N, Andersson G, Keiner AJ, Cacace AT, Martin N, Moore BC, Martin N. (2014) A review of hyperacusis and future directions: part I. Definitions and manifestations. Am J Audiol 23(4):402-419.

Vendrig AA. (2000) The Minnesota Multiphasic Personality Inventory and chronic pain: a conceptual analysis of a long-standing but complicated relationship. Clin Psychol Rev 20:533-559.

Walpurger V, Hebing-Lennartz G, Denecke H, Pietrowsky R. (2003) Habituation deficit in auditory event-related potentials in tinnitus complainers. Hear Res 181(1-2):57-64.

Weisz N, Voss S, Berg P, Elbert T. (2004) Abnormal auditory mismatch response in tinnitus sufferers with high-frequency hearing loss is associated with subjective distress level. BMC Neuroci 5(1):8.

Yochman A, Ornoy A, Parush S. (2006) Co-occurrence of developmental delays among preschool children with attention-deficithyperactivity disorder. Dev Med Child Neurol 48(6):483.

Zeng FG. (2013) An active loudness model suggesting tinnitus as increased central noise and hyperacusis as increased nonlinear gain. Hear Res 295:172-179. 